\title{
Investigation of nosocomial SARS-CoV-2 transmission from two patients to healthcare workers identifies close contact but not airborne transmission events
}

\author{
Derek J. Bays MD르, Minh-Vu H. Nguyen MD, MSc${ }^{1}$, Stuart H. Cohen MD¹, Sarah Waldman MD¹, Carla S. Martin MSN, RN², \\ George R. Thompson III MD ${ }^{1,3}$, Christian Sandrock MD, MPH ${ }^{4}$, Joel Tourtellotte MD, MSc ${ }^{4}$, Janelle Vu Pugashetti MD ${ }^{4}$, \\ Chinh Phan DO ${ }^{4}$, Hien H. Nguyen MD, MAS ${ }^{1,5}$, Gregory Y. Warner DO, MPH ${ }^{6, a}$ and Bennett H. Penn MD, PhD ${ }^{1,3 a}$ (i) \\ ${ }^{1}$ Division of Infectious Diseases, Department of Internal Medicine, University of California Davis Medical Center, Sacramento, California, ${ }^{2}$ Patient Care Services, \\ University of California Davis Medical Center, Sacramento, California, ${ }^{3}$ Department of Medical Microbiology and Immunology, University of California, Davis, \\ Davis, California, ${ }^{4}$ Division of Pulmonary, Critical Care, and Sleep Medicine, Department of Internal Medicine, University of California Davis Medical Center, \\ Sacramento, California, ${ }^{5}$ Division of Infectious Diseases, Department of Internal Medicine, Veterans' Affairs Northern California Health Care System, Sacramento, \\ California and ${ }^{6}$ Division of Infectious Diseases, Department of Internal Medicine, NorthBay Healthcare, Fairfield, California
}

\section{Abstract}

Objective: To describe the pattern of transmission of severe acute respiratory coronavirus virus 2 (SARS-CoV-2) during 2 nosocomial outbreaks of coronavirus disease 2019 (COVID-19) with regard to the possibility of airborne transmission.

Design: Contact investigations with active case finding were used to assess the pattern of spread from 2 COVID-19 index patients.

Setting: A community hospital and university medical center in the United States, in February and March, 2020, early in the COVID-19 pandemic.

Patients: Two index patients and 421 exposed healthcare workers.

Methods: Exposed healthcare workers (HCWs) were identified by analyzing the electronic medical record (EMR) and conducting active case finding in combination with structured interviews. Healthcare coworkers (HCWs) were tested for COVID-19 by obtaining oropharyngeal/ nasopharyngeal specimens, and RT-PCR testing was used to detect SARS-CoV-2.

Results: Two separate index patients were admitted in February and March 2020, without initial suspicion for COVID-19 and without contact or droplet precautions in place; both patients underwent several aerosol-generating procedures in this context. In total, $421 \mathrm{HCWs}$ were exposed in total, and the results of the case contact investigations identified 8 secondary infections in HCWs. In all 8 cases, the HCWs had close contact with the index patients without sufficient personal protective equipment. Importantly, despite multiple aerosol-generating procedures, there was no evidence of airborne transmission.

Conclusion: These observations suggest that, at least in a healthcare setting, most SARS-CoV-2 transmission is likely to take place during close contact with infected patients through respiratory droplets, rather than by long-distance airborne transmission.

(Received 30 April 2020; accepted 20 June 2020; electronically published 3 July 2020)

Multiple routes of transmission have been postulated for severe acute respiratory coronavirus virus 2 (SARS-CoV-2), including respiratory droplets, airborne particles, and fomites. ${ }^{1-4}$ In particular, the risk of acquiring coronavirus disease 2019 (COVID-19) through inhalation of airborne particles, capable of transmitting infection over long distances, is uncertain, and remains a matter of vigorous debate. ${ }^{2,5,6}$ Given the significant risks of transmission

Author for correspondence: Bennett Penn, E-mail: bhpenn@ucdavis.edu

${ }^{a}$ Authors of equal contribution.

Cite this article: Bays DJ, et al. (2021). Investigation of nosocomial SARS-CoV-2 transmission from two patients to healthcare workers identifies close contact but not airborne transmission events. Infection Control \& Hospital Epidemiology, 42: 1046-1052, https://doi.org/10.1017/ice.2020.321 to healthcare workers (HCWs), ${ }^{7}$ defining the degree to which airborne transmission occurs is important for guiding hospital infection control procedures and informing public health policy.

The predominant mode of transmission for most respiratory viruses occurs via large respiratory droplets inoculating mucous membranes. ${ }^{8}$ Respiratory droplets $>5 \mu \mathrm{m}$ in size travel $<2 \mathrm{~m}$, remain suspended $<20$ minutes, and are effectively blocked by surgical masks. ${ }^{8}$ In contrast, smaller droplets evaporate rapidly, and the remaining desiccated droplet nucleus can remain airborne for hours, travel long distances, and require N95 respirators for protection. $^{8}$

For SARS-CoV-2, several laboratory and environmental studies have suggested the possibility of airborne transmission. ${ }^{5,6,9}$ 
Artificially generated SARS-CoV-2 aerosols were found to be stable, with a half-life of $1.5 \mathrm{~h}$, and viral RNA has been detected on surfaces throughout the rooms of COVID-19 patients, including the ventilation system. The uncertain routes of transmission have led to inconsistent recommendations for infection prevention and appropriate personal protective equipment (PPE) for HCWs. For routine patient care, the WHO recommends contact and droplet precautions, ${ }^{4}$ and only recommends airborne precautions with a respirator in the setting of aerosol-generating procedures (AGPs). ${ }^{2}$ In contrast, the US Centers for Disease Control and Prevention (CDC) expresses a preference for respirators as routine PPE, with droplet and contact precautions being considered an acceptable alternative in the context of supply shortages. ${ }^{3}$

To assess the routes of transmission of SARS-CoV-2, it will be necessary to document the pattern of spread from well-defined exposures. Here, we describe the pattern of nosocomial SARSCoV-2 transmission from 2 separate patients who were not initially suspected as having COVID-19 and who were cared for without contact, droplet, or airborne precautions.

\section{Methods}

\section{Contact investigations}

Investigation $1 \mathrm{~A}$ reviews the contact investigation for patient 1 at hospital A, a community hospital. The HCWs wore neither surgical masks nor eye protection, and they were risk stratified based on examination of the medical record and subsequent phone interviews as follows: high risk: nose or mouth exposed during intubation or bronchoscopy; moderate: nose or mouth exposed for $>2$ minutes; and low: nose or mouth exposed $<2$ minutes.

Investigation $1 \mathrm{~B}$ and investigation 2 were completed at hospital $\mathrm{B}$, a university medical center. In both instances, hospital B undertook active case finding, with a combination of electronic medical record (EMR) tracing to identify all HCWs who entered the index patient's record, as well as surveys conducted by each unit manager to identify any HCWs that may have entered the room without EMR contact. Exposed HCWs filled out structured surveys regarding PPE, and types of contact, including AGPs. In addition, any HCWs with an influenza-like illness (ILI) underwent testing as per hospital policy, including HCWs not directly involved in the patient's clinical care. A number of asymptomatic HCWs were also tested because they were deemed higher risk for transmitting to patients, including respiratory therapists and all members of the oncology team.

Exposed HCWs at hospital B were risk stratified with the following designations: patient source controlled with mask or intubation $(\mathrm{Con}+/-)$, and PPE with surgical mask $(\mathrm{M}+/-)$ and eye protection $(\mathrm{E}+/-)$. Risk level was defined as: high: (Con-M-E-), moderate (Con-M-E+ or Con-M+E-), and low (Con-M+E+ or $\mathrm{Con}+\mathrm{M}+\mathrm{E}-$ ). No asymptomatic patients were tested, and no patients developed an ILI that triggered SARS-CoV-2 testing. Using the criteria of hospital B, all exposures at hospital A would have been considered high risk because no HCWs wore masks, eye protection, or gowns. For case 1, testing relied on oropharyngeal or nasopharyngeal swabs, with RT-PCR performed at the California Department of Public Health (CADPH). For case 2, only nasopharyngeal swabs were used, and specimens were tested on-site by hospital B using a validated assay on an ABI StepOnePlus instrument. The institutional review board (IRB) at hospital B deemed that IRB approval and informed consent were unnecessary due to the quality improvement origins of the work.

\section{Statistical analysis}

The Fisher exact test was used to assess the association between specific high-risk procedures and a positive SARS-CoV-2 using RT-PCR.

\section{Case 1}

\section{Clinical course at hospital $A$}

A previously healthy woman aged in her 40 s, who would later be deemed the first case of community-acquired COVID-19 diagnosed in the United States, presented to a local hospital with 48 hours of subjective fever, dry cough, nausea, and vomiting. ${ }^{10}$ Upon presentation she was febrile, tachycardic, and hypotensive, and her chest $\mathrm{x}$-ray showed a focal consolidation. The patient was admitted to the general medical-surgical ward with a diagnosis of community-acquired pneumonia, and intravenous antibiotics treatment began. Over 2 days, she became increasingly hypoxic, requiring oxygen through high-flow nasal canula, and her chest $\mathrm{x}$-ray showed progressive disease. By day 3 , she required noninvasive mechanical ventilation, and she eventually transferred to the intensive care unit (ICU) and endotracheally intubated. The patient underwent an initial abbreviated bronchoscopy followed by a second longer diagnostic bronchoscopy that showed only bloody secretions. The patient's hypoxemia worsened, and she was subsequently transferred on day 4 to hospital B for consideration of extracorporeal membrane oxygenation (ECMO).

\section{Clinical course after transfer to hospital B}

Extensive additional evaluation for the etiology of acute respiratory distress syndrome (ARDS) was completed and unrevealing. Despite broad-spectrum antimicrobials, she had persistent fever and hypoxemic respiratory failure, although she did not require ECMO. At the time of her illness, SARS-CoV-2 testing was only available through the CDC. Although she had no travel history that qualified her for SARS-CoV-2 testing, her severe presentation and unrevealing diagnostic evaluation suggested the possibility of COVID-19. On day 5 of her course at hospital B, a nasopharyngeal swab for SARS-CoV-2 was sent to the CDC, which returned positive. Remdesivir was obtained through a compassionate use authorization, the patient slowly improved, and ultimately, was discharged home after $\sim 1$ month.

\section{Case 1 contact investigation-hospital A}

Because COVID-19 was not initially suspected, a large number of HCWs was exposed to the index case at hospital A without PPE; many of the details of this contact investigation have been recently reported in a CDC-issued Morbidity and Mortality Weekly Report. ${ }^{11}$ No contact, droplet, or airborne precautions were used. The patient initially spent time on the combined medicalsurgical ward with 29 beds and staffed by 8 RNs, several physicians, and ancillary staff. She was then was transferred to the ICU, which has 6 rooms. The patient's room for intubation and bronchoscopy measures $22.76 \mathrm{~m}^{2}$ (245 feet ${ }^{2}$ ), was not negative pressure, and has net air exchange of $2.83 \mathrm{~m}^{3} / \mathrm{min}\left(100 \mathrm{feet}^{3} / \mathrm{min}\right)$.

The contact investigation at hospital A determined that, in total, 126 HCWs were exposed, of whom 28 HCWs were deemed high risk, 67 were deemed moderate risk, and 31 were deemed low risk. Of these $126 \mathrm{HCWs}, 43$ developed an ILI. These 43 HCWs were tested, and 3 HCWs tested positive (Fig. 1). All 3 had provided direct patient care with close contact for several days, and were 


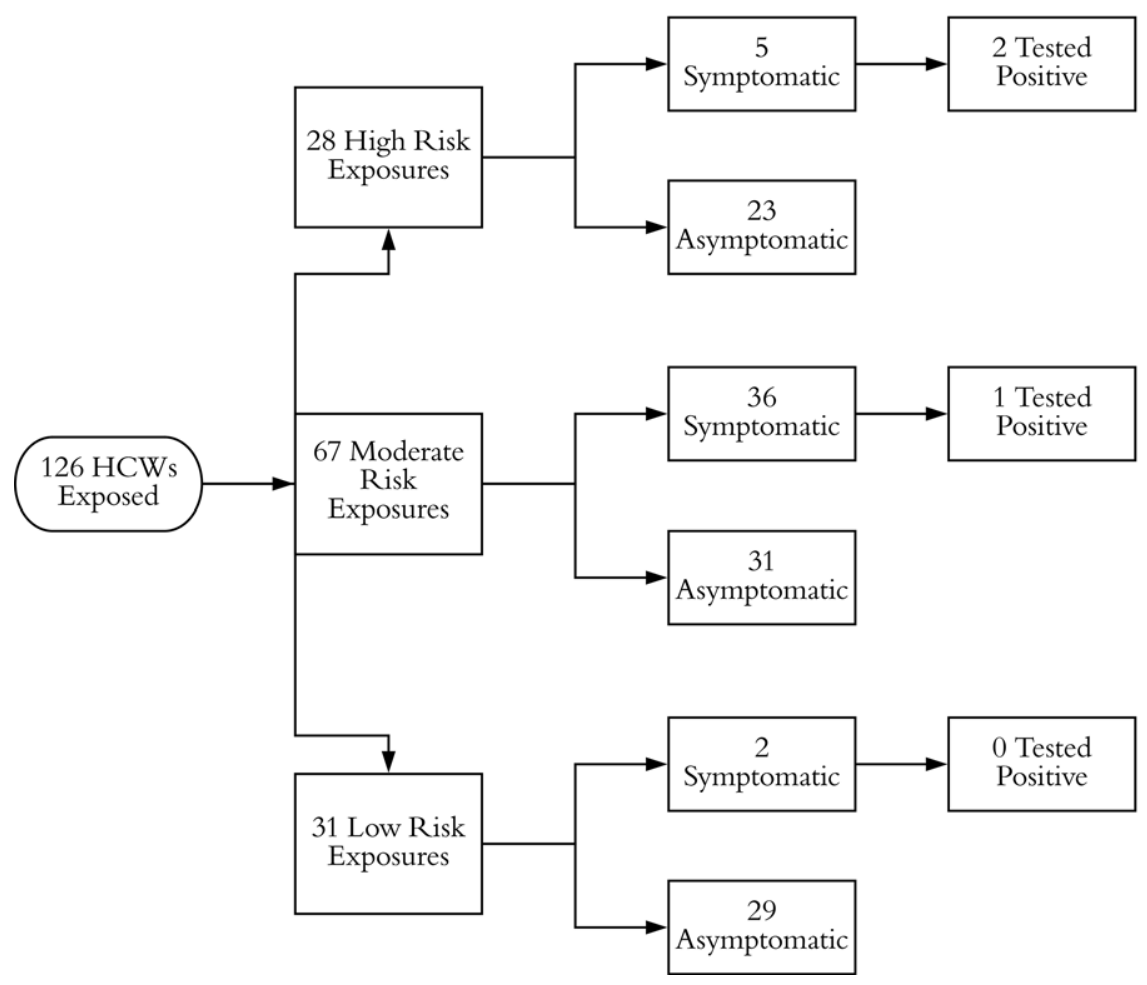

Fig. 1. Stratification of exposed healthcare workers (HCWs) for case 1 at hospital A.
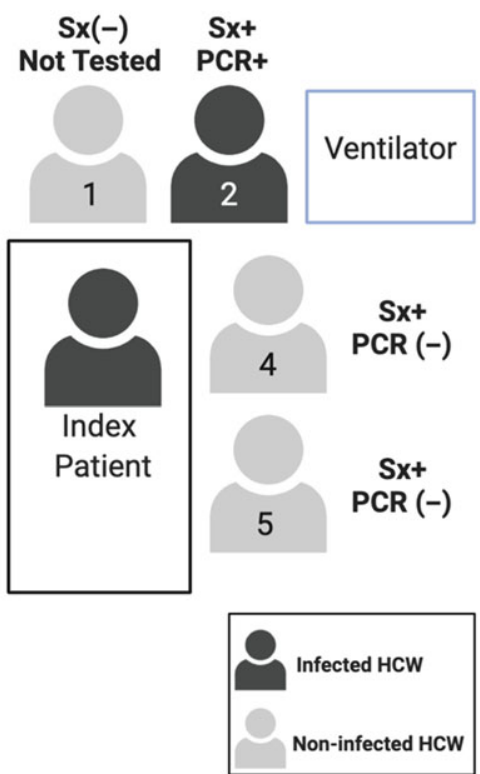

Fig. 2. Description of staff at endotracheal intubation for case 1 at hospital A. Note. $\mathrm{Sx}+$, symptomatic; Sx (-) asymptomatic; PCR +/(-) denotes SARS-CoV-2 test result.

present for AGPs without masks or eye protection. Two SARS$\mathrm{CoV}$-2-positive HCWs were direct providers on the ward and were present while the patient received oxygen by high flow nasal cannula or noninvasive positive-pressure ventilation, the third HCW provided care both on the medical ward and the ICU and was present for the intubation and bronchoscopies (Fig. 2). In summary, although $43 \mathrm{HCWs}$ underwent testing, the infected HCWs all had had prolonged direct contact with the patient, including during AGPs.

\section{Case 1 contact investigation-hospital B}

Upon transfer, droplet and contact precautions were instituted, and respiratory pathogen PCR testing was performed. On hospital day 3, when the respiratory pathogen panel returned negative, droplet and contact precautions were discontinued. On hospital day 5 , when it became possible to test for SARS-CoV-2, airborne precautions were instituted. Testing returned positive, and airborne precautions were continued until hospital day 23, when she had 2 negative SARS-CoV-2 nasopharyngeal swabs from consecutive days return negative, and airborne, droplet, and contact precautions were discontinued. Prior to the institution of airborne isolation, $147 \mathrm{HCWs}$ at hospital B were exposed to the patient (Fig. 3). There were 15 high-risk exposures, 73 mediumrisk exposures, and 59 low-risk exposures. All of the high- and medium-risk HCWs (88 HCWs) were isolated from work for 14 days. Ultimately, 13 employees developed ILI symptoms and were tested for SARS CoV-2 using RT-PCR, but all tested negative.

\section{Case 2}

\section{Clinical course}

A previously healthy man aged in his 60 s presented to a local hospital with dyspnea. He was found to have a deep vein thrombosis with pulmonary emboli and was noted to have a leukocyte cell count of 69,000 cells $/ \mathrm{mm}^{3}$ with myeloblasts. He was transferred to hospital B, and on day 2, he developed progressive hypoxemic respiratory failure; he was intubated on day 3 . Bone marrow biopsy confirmed AML, and his course was complicated by the presumed sequalae of leukostasis with disseminated intravascular coagulation, acute left middle cerebral artery infarct, subarachnoid hemorrhage, acute kidney injury, and splenic rupture. He remained persistently febrile and underwent an unrevealing diagnostic bronchoscopy, and on day 14 the infectious diseases service was consulted. A nasopharyngeal swab for SARS-CoV-2 testing was 
Fig. 3. Stratification of exposed healthcare workers (HCWs) for case 1 at hospital $B$.

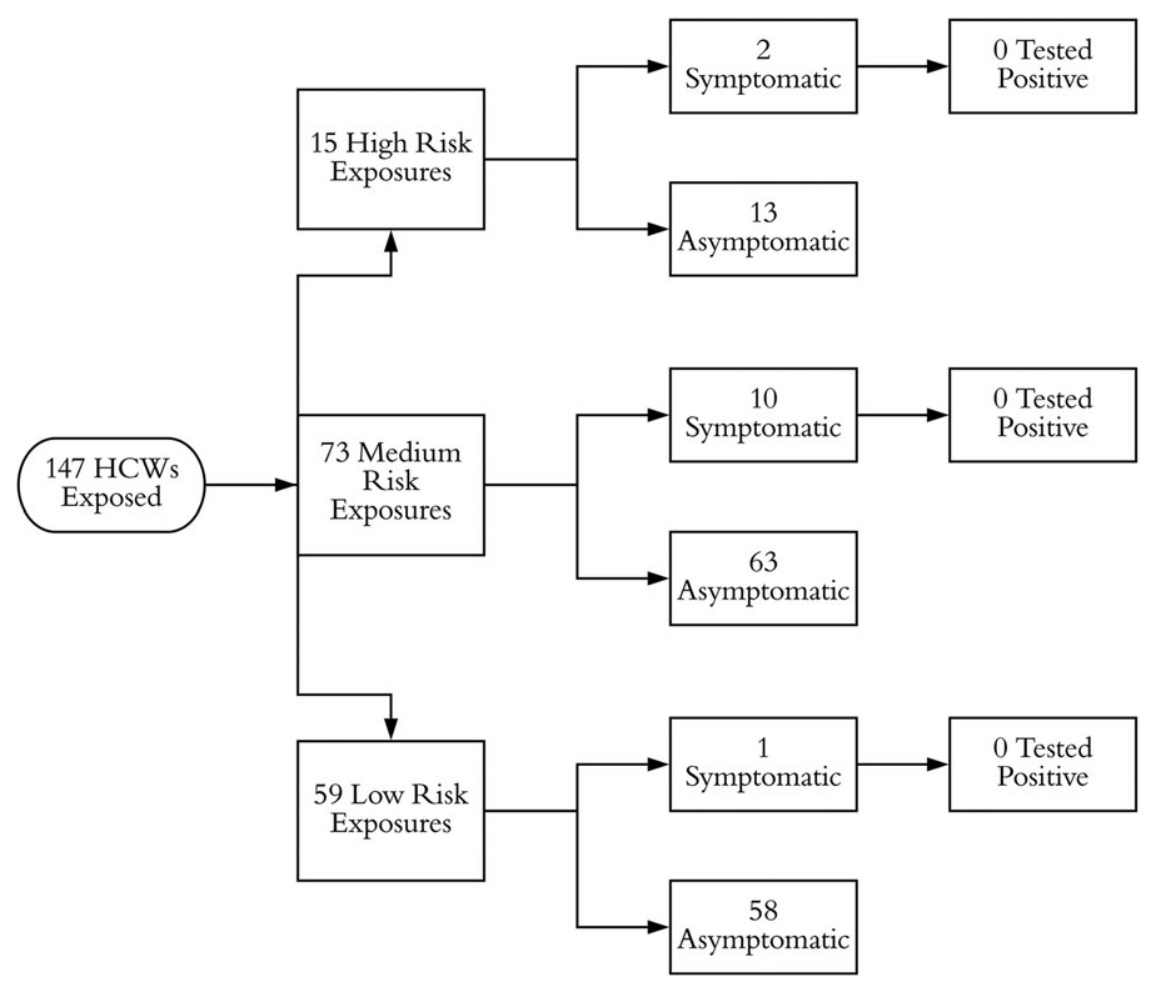

obtained, and droplet and contact precautions, with airborne precautions for AGPs were instituted. SARS-CoV-2 testing returned positive on day 15 , with a cycle-threshold $(\mathrm{Ct})$ value of 25 . The patient then developed central venous catheter-associated bloodstream infection with septic shock and despite intravenous antibiotics and catheter removal, he continued to decline. The family ultimately opted to pursue comfort-focused care, and the patient died on hospital day 30 .

\section{Case 2 contact investigation}

The patient was originally admitted to the oncology unit, which consists of 25 neutral-pressure rooms with 35 beds and is staffed by 13 nurses per shift and 5-6 physicians on the oncology team. He was transferred to the medical ICU, which consists of 16 single-room beds, each with an assigned nurse, as well as 2 teams with 6-7 physicians each and a variable number of respiratory therapists. The room in which the patient was intubated measures $15.33 \mathrm{~m}^{2}$ (165 feet ${ }^{2}$ ) and has 15 air exchanges per hour but is not negative pressure relative to the unit. Between hospital day 3 and day 15 , there was some degree of source control; his ventilator was fitted with closed-circuit suctioning an in-line high-efficiency particulate air (HEPA) filter.

Overall, 145 HCWs were identified as having exposure to the index patient, with 5 confirmed infections and 2 possible infections (Fig. 4). Of the $145 \mathrm{HCWs}, 7$ developed ILI symptoms and all were at the bedside for AGPs without adequate PPE. The patient underwent 2 significant AGPs: endotracheal intubation on day 3 and bronchoscopy on day 11, with neither airborne nor droplet precautions in place. Most transmission events were associated with the endotracheal intubation; 4 of the $7 \mathrm{HCWs}$ present for the procedure tested positive for SARS-CoV-2 $(P<.001)$ (Fig. 5). The individual performing the procedure wore a surgical mask without eye protection, and the remaining HCWs wore neither masks nor eye protection (Table 1). A fifth HCW, who was at neither the bronchoscopy nor intubation, also developed symptoms, but this HCW had direct patient contact for several days without PPE and assisted in transferring the patient between ventilators, which necessitated a break in the closed ventilation circuit. All HCWs who tested positive developed symptoms within a 72-hour window. Two additional HCWs who had direct patient contact without PPE during AGPs, whom we consider possible cases, developed high fevers and cough but tested negative for SARSCoV-2 twice each. Interestingly, we identified no transmission during the bronchoscopy when all HCWs wore surgical masks and eye protection. Overall 7 HCWs were present, and the 2 providers who performed the bronchoscopy wore surgical masks with eye protection, a gown, and gloves. Everyone else wore a surgical mask with eye protection (Table 1). Thus, in summary, although a number of HCWs became infected by the index case, they all had direct contact with the patient and were present during AGPs without sufficient PPE.

\section{Discussion}

The cases described here, and the pattern of spread to exposed HCWs, provide important insight into the transmissibility of SARS-CoV-2 in a healthcare setting. Both patients were in the hospital for several days before COVID-19 was suspected, without contact, droplet, or airborne precautions in place, and both patients underwent multiple AGPs without negative-pressure isolation rooms. The hypothesis that SARS-CoV-2 is airborne transmissible would predict widespread infection of HCWs or other patients during this time, unconstrained by the $2-\mathrm{m}$ radius that large respiratory droplets travel. Indeed, this is precisely the pattern seen with well-established airborne-transmitted agents such as tuberculosis and measles, in which patients cared for without negative-pressure isolation have triggered multiple outbreaks, with 


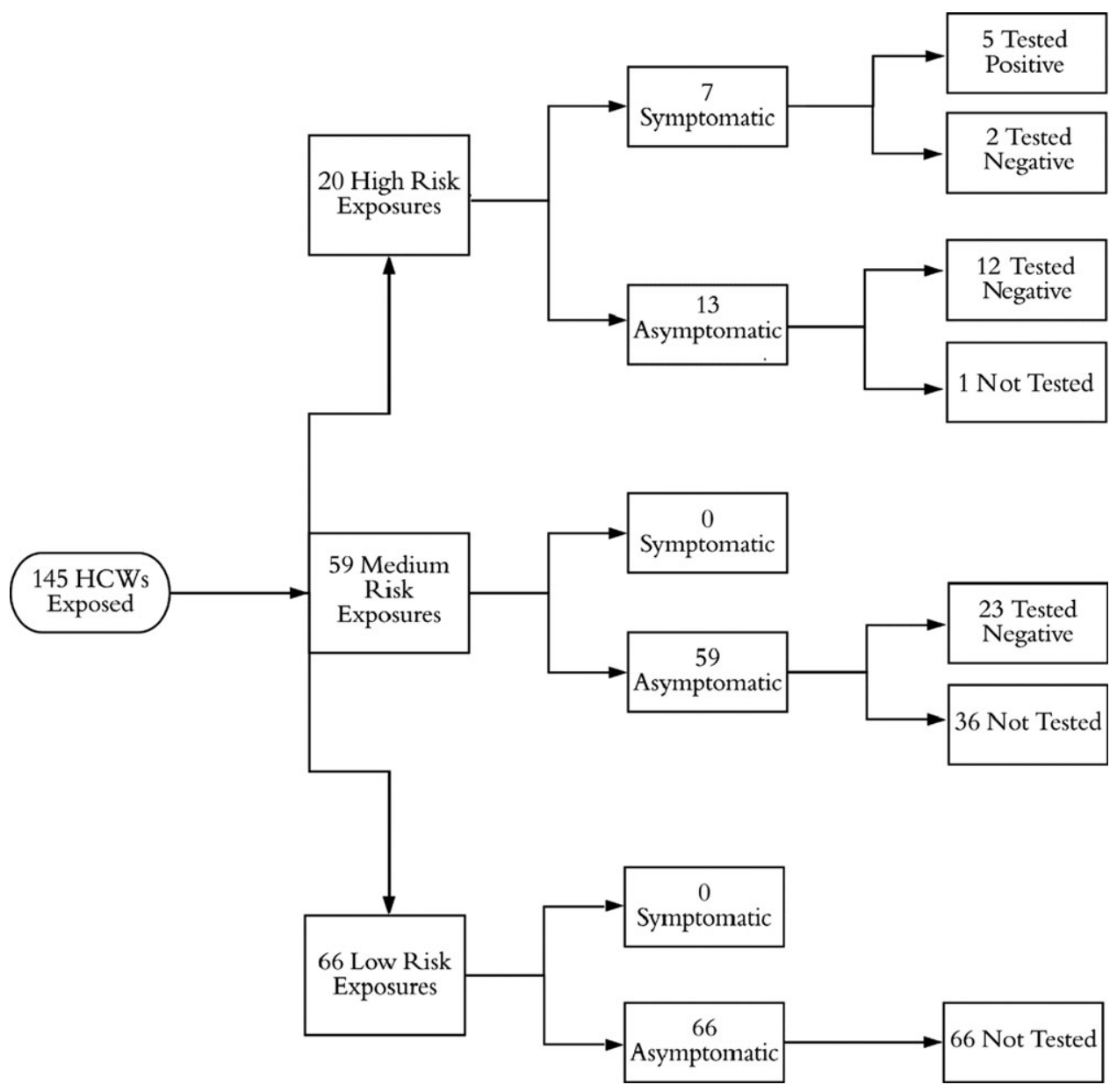

Fig. 4. Stratification of exposed healthcare workers (HCWs) for case 2.

infection spreading to HCWs and other patients throughout a unit who had no direct contact with the index case. ${ }^{13-15}$

For the cases described here, this did not occur. Although 8 HCWs were infected, transmission occurred exclusively among HCWs that were at the patient's bedside without contact and droplet PPE. No apparent transmission to HCWs or patients occurred elsewhere on the units, including an oncology ward housing a large number of immunocompromised patients. These findings are much more consistent with transmission by respiratory droplets than by airborne transmission. ${ }^{16,17}$ This idea is further supported by 3 other recent reports, each of individual patients with unsuspected COVID-19 in which an absence of airborne transmission was similarly documented. ${ }^{18-20}$

Several possibilities exist to reconcile the theoretical concern for airborne transmission raised by other studies ${ }^{5,6,21}$ with our contact investigations showing no apparent airborne transmission. Although artificially generated SARS-CoV-2 airborne particles are quite stable and SARS-CoV-2 RNA can be detected throughout COVID-19 patient rooms, no infectious virus could be recovered from the rooms, suggesting that the viral RNA might be from replication intermediates or noninfectious virions. However, it is impossible to exclude the possibility that virions isolated from surfaces were initially infectious but had degraded prior to sample collection. An alternative explanation may lie in the dose of SARS-CoV-2 necessary to establish infection. The minimum infectious dose varies dramatically between respiratory pathogens, with $<10$ bacilli needed to establish $M$. tuberculosis infection, but $>500$ virions needed for echovirus. ${ }^{20}$ The minimum infectious dose for
SARS-CoV-2 in humans is unknown, but a likely explanation for a failure of SARS-CoV-2 airborne particles to efficiently transmit infection over long distances may simply be that the number of inhaled virions is insufficient to establish infection.

Our observations did not discriminate between close-range transmission by large respiratory droplets that can be effectively blocked by surgical masks and eye protection versus close-range transmission by small droplets and droplet nuclei that penetrate surgical masks. The potential importance of these small particles was highlighted during the 2003 SARS outbreak, when high-risk AGPs such as intubation and cardiopulmonary resuscitation were analyzed. During these procedures, HCWs with close patient contact became infected, even when droplet precautions were in place, with endotracheal intubation having an odds ratio of $13 .{ }^{21}$ Because the HCWs who became infected in our study wore neither droplet nor airborne protective equipment, we cannot assess the relative degree of protection that would have been provided by droplet precautions relative to N95 respirators.

Our report has several limitations. Most importantly, a large number of HCWs was exposed, with 421 individuals identified by contact tracing, we were limited by having only 2 index cases, and we had viral load information for only 1 case. This patient had a Ct value of 25, approximately the median value of other studies. ${ }^{24}$ Possibly, patients with higher viral loads more readily transmit infection via airborne particles. In addition, at the time these hospital outbreaks occurred, in February and March 2020, testing infrastructure was still very limited, and systematic testing of all asymptomatic HCWs or patients on each unit was not possible. 
Table 1. Summary of PPE and SARS-CoV-2 Testing for Case 2 Procedures

\begin{tabular}{|c|c|c|}
\hline \multicolumn{3}{|c|}{ Intubation } \\
\hline Staff 1 & Gloves, no mask or eye protection & Symptomatic, PCR + \\
\hline Staff $2^{\mathrm{a}}$ & $\begin{array}{l}\text { Gloves and mask without eye } \\
\text { protection }\end{array}$ & Symptomatic, PCR + \\
\hline Staff 3 & Gloves, no mask or eye protection & Symptomatic, PCR + \\
\hline Staff 4 & Gloves, no mask or eye protection & Symptomatic, PCR + \\
\hline Staff 5 & Gloves, no mask or eye protection & $\begin{array}{l}\text { Symptomatic, } \\
\text { PCR }(-) \times 2\end{array}$ \\
\hline Staff 6 & Gloves, no mask or eye protection & $\begin{array}{l}\text { Asymptomatic, not } \\
\text { tested }\end{array}$ \\
\hline Staff 7 & Gloves, no mask or eye protection & $\begin{array}{l}\text { Asymptomatic, not } \\
\text { tested }\end{array}$ \\
\hline \multicolumn{3}{|c|}{ Bronchoscopy } \\
\hline Staff $7^{a}$ & Mask, eye protection, gown, gloves & $\begin{array}{l}\text { Asymptomatic, not } \\
\text { tested }\end{array}$ \\
\hline Staff $8^{a}$ & Mask, eye protection, gown, gloves & $\begin{array}{l}\text { Asymptomatic, not } \\
\text { tested }\end{array}$ \\
\hline Staff 9 & Mask, eye protection & Symptomatic, PCR (-) \\
\hline Staff 10 & Mask, eye protection & $\begin{array}{l}\text { Asymptomatic, not } \\
\text { tested }\end{array}$ \\
\hline Staff 11 & Mask, eye protection & $\begin{array}{l}\text { Asymptomatic, not } \\
\text { tested }\end{array}$ \\
\hline Staff 12 & Mask, eye protection & $\begin{array}{l}\text { Asymptomatic, not } \\
\text { tested }\end{array}$ \\
\hline
\end{tabular}

Note. Mask indicates surgical mask; PCR $+/(-)$ denotes SARS-CoV-2 test result. ${ }^{a}$ Denotes staff performing the procedure.
Given the likelihood of asymptomatic COVID-19 cases, ${ }^{25}$ we cannot exclude occult transmission leading to asymptomatic secondary cases. However, no additional cases were detected amongst the 35 asymptomatic HCWs who were able to be tested, and all HCWs who developed ILI symptoms were tested for SARS$\mathrm{CoV}-2$, regardless of whether they had had contact with an index patient. Finally, the sensitivity of a single nasopharyngeal test has been reported at $63 \%,{ }^{22}$ so some HCWs with COVID-19 may have gone undetected. However, limitations in test sensitivity would apply to HCWs both with and without direct contact and would not be expected to bias the overall distribution of cases. In summary, our findings suggest that, at least in a healthcare setting, most SARS-CoV-2 transmission likely takes place during close contact with infected patients rather than by long-distance airborne transmission.

\section{Acknowledgments.}

Financial support. No financial support was provided relevant to this article.

Conflicts of interest. All authors report no conflicts of interest relevant to this article.

\section{References}

1. Ferioli M, Cisternino C, Leo V, Pisani L, Palange P, Nava S. Protecting healthcare workers from SARS-CoV-2 infection: practical indications. Eur Respir Rev 2020;29(155):200068. doi: 10.1183/16000617.0068-2020.

2. Rational use of personal protective equipment for coronavirus disease 2019 (COVID-19) interim guidance. World Health Organization website. https://apps.who.int/iris/bitstream/handle/10665/331498/WHO-2019-nCoVIPCPPE_use-2020.2-eng.pdf?sequence=1\&isAllowed=y. Published 2020. Accessed 6 June, 2020.

3. Coronavirus disease 2019 (COVID-19) infection control guidance. Centers for Disease Control and Prevention website. https://www.cdc.gov/ coronavirus/2019-ncov/hcp/infection-control-recommendations.html. Published 2020. Accessed June 6, 2020.

4. Organization WH. Modes of transmission of virus causing COVID-19: implications for IPC precaution recommendations. Centers for Disease Control and Prevention website. https://apps.who.int/iris/bitstream/ handle/10665/331601/WHO-2019-nCoV-Sci_Brief-Transmission_modes2020.1-eng.pdf. Published 2020. Accessed June 6, 2020.

5. van Doremalen N, Bushmaker T, Morris DH, et al. Aerosol and surface stability of SARS-CoV-2 as compared with SARS-CoV-1. N Engl J Med 2020; 382:1564-1567.

6. Guo ZD, Wang ZY, Zhang SF, et al. Aerosol and surface distribution of severe acute respiratory syndrome coronavirus 2 in hospital wards, Wuhan, China, 2020. Emerg Infect Dis 2020;26.

7. Team CC-R. Characteristics of Health Care Personnel with COVID-19United States, February 12-April 9, 2020. Morb Mortal Wkly Rep 2020; 69:477-481.

8. Kutter JS, Spronken MI, Fraaij PL, Fouchier RA, Herfst S. Transmission routes of respiratory viruses among humans. Curr Opin Virol 2018; 28:142-151.

9. Santarpia JL, Rivera DN, Herrera V, et al. Aerosol and surface transmission potential of SARS-CoV-2. medRxiv 2020 June 3. doi: 10.1101/2020.03.23. 20039446.

10. Sanville B, Corbett R, Pidcock W, et al. A community transmitted case of severe acute respiratory distress syndrome due to SARS CoV2 in the United States. Clin Infect Dis 2020 March 30 [Epub ahead of print]. doi: 10.1093/ cid/ciaa347.

11. Heinzerling A, Stuckey MJ, Scheuer T, et al. Transmission of COVID-19 to healthcare personnel during exposures to a hospitalized patient-Solano County, California, February 2020. Morb Mortal Wkly Rep 2020;69:472-476.

12. Cheung JC, Ho LT, Cheng JV, Cham EYK, Lam KN. Staff safety during emergency airway management for COVID-19 in Hong Kong. Lancet Respir Med 2020;8:e19.
Fig. 5. Description of staff present during intubation of case 2. Note. Sx+, symptomatic; Sx(-) asymptomatic; PCR $+/(-)$ denotes SARS-CoV-2 test result. 
13. Pearson ML, Jereb JA, Frieden TR, et al. Nosocomial transmission of multidrug-resistant Mycobacterium tuberculosis: a risk to patients and healthcare workers. Ann Intern Med 1992;117:191-196.

14. Sienko DG, Friedman C, McGee HB, et al. A measles outbreak at university medical settings involving health care providers. Am J Public Health 1987;77:1222-1224.

15. Rivera ME, Mason WH, Ross LA, Wright HT Jr. Nosocomial measles infection in a pediatric hospital during a community-wide epidemic. J Pediatr 1991;119:183-186.

16. Poutanen SM, Low DE, Henry B, et al. Identification of severe acute respiratory syndrome in Canada. N Engl J Med 2003;348:1995-2005.

17. Seto WH, Tsang D, Yung RW, et al. Effectiveness of precautions against droplets and contact in prevention of nosocomial transmission of severe acute respiratory syndrome (SARS). Lancet 2003;361:1519-1520.

18. Ng K, Poon BH, Kiat Puar TH, et al. COVID-19 and the risk to healthcare workers: a case report. Ann Intern Med 2020;172:766-767.

19. Wong SCY, Kwong RT, Wu TC, et al. Risk of nosocomial transmission of coronavirus disease 2019: an experience in a general ward setting in Hong Kong. J Hosp Infect 2020;105:119-127.
20. Schwierzeck V, Konig JC, Kuhn J, et al. First reported nosocomial outbreak of severe acute respiratory syndrome coronavirus 2 (SARS-CoV-2) in a pediatric dialysis unit. Clin Infect Dis 2020 Apr 27 [Epub ahead of print]. doi: 10.1093/cid/ciaa491.

21. Liu Y, Ning Z, Chen Y, et al. Aerodynamic analysis of SARS-CoV-2 in two Wuhan hospitals. Nature 2020;582:557-560.

22. Ward RL, Akin EW, D’Alessio DJ. Minimum infective dose of animal viruses. Crit Revs Environ Control 1984;14:297-310.

23. Fowler RA, Guest CB, Lapinsky SE, et al. Transmission of severe acute respiratory syndrome during intubation and mechanical ventilation. Am J Respir Crit Care Med 2004;169:1198-1202.

24. Wang W, Xu Y, Gao R, et al. Detection of SARS-CoV-2 in different types of clinical specimens. JAMA 2020;323:1843-1844.

25. Mizumoto K, Kagaya K, Zarebski A, Chowell G. Estimating the asymptomatic proportion of coronavirus disease 2019 (COVID-19) cases on board the Diamond Princess cruise ship, Yokohama, Japan, 2020. Euro Surveill 2020;25:2000180. doi: 10.2807/1560-7917.ES.2020.25. 10.2000180 